provincial policy and programs differ greatly in availability and cost of PrEP, use of PrEP increased in all sites during the course of the study.

Disclosure No significant relationships.

\section{P504 REDUCTION OF THE SPREAD OF HIV AMONG MEN WHO HAVE SEX WITH MEN IN BAMENDA-CAMEROON}

Assi Flaviurs. Vicony's Global Success World, Public Health, Bamenda, Cameroon

10.1136/sextrans-2019-sti.584

Background In 2010, i conducted a study that aimed to produce information on the magnitude and characteristics of the HIV epidemic in the MSM population of 18 years or more, and sexual behavior in two prison and region of the country between these region is Bamenda, located in the north west of the country with an estimated population of 2.5 million. Alternative Cameroon and Vicony's Global Success World (VGSW) in Bamenda was responsible for the execution of the study and was responsible for the protocol provided.

Methods

Data collection was carried out by the method of sampling conducted by respondents (Respondent Driven Sampling RDS) and the application of a serological survey anonymous linked. The national sample consisted of 200 MSM in the region of Bamenda by 333 .

Results HIV prevalence $8.1 \%$ in North West region (Ministry of public health 2010).

The $0 \%$ are considered bisexual, homosexual $75.2 \%, 1.8 \%$ heterosexual.

$10.8 \%$ of MSM in the region do not know where to go if they want to get tested for HIV.

21.9\% underwent a test for HIV in the last 12 months, and $50.3 \%$ received advice.

Only $10.6 \%$ knew that condoms can be achieved in health institutions.

28.1\% had to fake his sexual orientation.

Conclusion The results show a flaw in the processes of prevention and education for HIV and STIs among MSM and experience has served the Civil Society Organizations and the Government of the region to find a way to solve the problems that are evident in the response.

Clearly the stigma and discrimination in the area of HIV associated with MSM.

Bamenda could not be completed sample was estimated that 350 people (only reached 333) due to homophobia's attack project site and where material were destroyed, and tracking documents.

This study encourages us to move forward despite the adversities and difficulties.

Disclosure No significant relationships.

\section{P505 THE IMPACT OF GONORRHOEA VACCINATION IN MEN WHO HAVE SEX WITH MEN ON PREVALENCE AND RESISTANCE: MATHEMATICAL MODELLING STUDY}

\footnotetext{
${ }^{1}$ Janneke Heijne*, ${ }^{1}$ Maria Xiridou, ${ }^{2}$ Katy Turner, ${ }^{1}$ Birgit Van Benthem, ${ }^{3}$ Nicola Low. ${ }^{1}$ National Institute for Public Health and the Environment (RIVM), Epidemiology and Surveillance, Centre for Infectious Diseases Control, Bilthoven, Netherlands; ${ }^{2}$ University of Bristol, Bristol, UK; ${ }^{3}$ Institute of Social and Preventive Medicine (ISPM), Bern, Switzerland
}

10.1136/sextrans-2019-sti.585
Background Men who have sex with men (MSM) are disproportionately affected by Neisseria gonorrhoeae (gonorrhoea) and antimicrobial resistance (AMR) is common. Gonorrhoea vaccine development is challenging, but a N. meningitidis (group B) vaccine might have reduced gonorrhoea incidence by $\approx 30 \%$. This study aims to investigate the impact of vaccination on gonorrhoea transmission and on AMR in MSM.

Methods We developed a deterministic compartmental model of gonorrhoea transmission among Dutch MSM. We included three sexual activity classes that differed in numbers of partners/year and unprotected sex acts/partnership. AMR to firstline treatment was assumed to be complete, occurring at a low probability after treatment. We modelled a partially protective prophylactic vaccine (reducing susceptibility) providing 2 years' protection. We estimated the prevalence of sensitive and resistant gonorrhoea strains after 10 and 50 years for vaccine efficacies (VE) of 30\% (lower) and 60\% (higher) and different uptake levels in high sexual activity MSM.

Results Gonorrhoea prevalence in the model was $0.45 \%$ overall, $8.3 \%$ in high sexual activity MSM, and $0.002 \%$ resistant strains (baseline). With lower VE, after 10 years, the prevalence of the sensitive strain was reduced by $75 \%$ (for $50 \%$ uptake). AMR prevalence increased (irrespective of uptake) although less than without vaccination. With lower VE, after 50 years, AMR prevalence decreased only with uptake $\geq 60 \%$, levels at which the sensitive strain was already eliminated. With higher VE, AMR prevalence decreased after 10 years for uptake $\geq 30 \%$ and after 50 years for uptake of $\geq 20 \%$.

Conclusion In this modelling study, a partially protective prophylactic vaccine for gonorrhoea decreased overall prevalence, even with lower VE. This study also shows how vaccination can reduce the spread of AMR. For gonorrhoea, vaccination can decrease the speed at which AMR is transmitted, and even reduce AMR prevalence, but only with higher levels of vaccine efficacy or uptake in high sexual activity MSM.

Disclosure No significant relationships.

\section{P506 OPTIMIZING EPT FOR MSM AND TRANSGENDER WOMEN}

${ }^{1}$ Kristi Gamarel, ${ }^{2}$ Raha Richardson*, ${ }^{2}$ Rob Stephenson, ${ }^{2}$ Okeoma Mmeje. ${ }^{1}$ University of Michigan, Health Behavior and Health Education, Ann Arbor, USA; ${ }^{2}$ University of Michigan, Ann Arbor, USA

\section{$10.1136 /$ sextrans-2019-sti.586}

Background Despite increasing rates of HIV and other STIs among men who have sex with men (MSM) and transgender women (TW), expedited partner therapy (EPT) is not routinely offered to these priority populations. The goal of this study was to understand healthcare providers' (HCP) views and opinions regarding the use of EPT with MSM and TW. Methods Between May and October 2018, 18 HCP in Michigan completed semi-structured interviews. The interviews covered questions about knowledge, attitudes, experiences with, and barriers and strategies for delivering EPT to MSM and TW.

Results HCP $\left(\right.$ Mean $\left._{\mathrm{age}}=47.4\right)$ included MDs (33\%), NPs (61\%), RNs (5\%), and a Pharmacist (1\%). The majority of HCP (94.5\%) were willing to prescribe EPT for chlamydia and gonorrhea to MSM and TW. Several HCP in community health clinics were currently prescribing EPT to MSM and TW, whereas others followed CDC guidelines. Some HCP expressed concerns about the provision of EPT for individuals 
with multiple sexual partners, persistent/recurrent infection, and potential antimicrobial resistance; however, none of the HCP believed there was a difference in efficacy based on a client's sexual preferences or gender identity. HCP who provided EPT described strategies to overcome these barriers, such as capitalizing on pharmacies and phone calls or videoconference calls with patients' partners to discuss potential allergies, treatment regimens, and ensuring linkage to HIV testing. HCP described how telemedicine and the use of peer health navigators could help overcome systemic barriers to regular STI testing (e.g., transportation, delayed appointment times) and potentially overcome medical mistrust around HIV prevention and care.

Conclusion The provision of EPT represents an overlooked yet important strategy to curb increasing STI rates among MSM and TW. Changing EPT legislation and CDC guidelines to include MSM and TW represents a promising avenue to link these communities to HIV and other STI prevention services. Disclosure No significant relationships.

\section{P507 DISCLOSURE OF SAME-SEX BEHAVIOR AND EXTRAGENITAL STI TESTING AMONG MEN WHO HAVE SEX WITH MEN - AMERICAN MEN'S INTERNET SURVEY}

${ }^{1}$ Alex De Voux* ${ }^{2}$ Kyle Bernstein, ${ }^{2}$ Robert Kirkcaldy, ${ }^{3}$ Maria Zlotorzynska, ${ }^{3}$ Travis Sanchez. ${ }^{1}$ CDC, Division of STD Prevention, Atlanta, USA; ${ }^{2}$ Centers for Disease Control and Prevention, Atlanta, USA; ${ }^{3}$ Emory University Rollins School of Public Health, Department of Epidemiology, Atlanta, USA

\subsection{6/sextrans-2019-sti.587}

Background Current guidelines recommend that sexually active men who have sex with men (MSM) be screened at least annually for bacterial sexually transmitted infections (STI) at sites of sexual contact regardless of condom use. Extragenital (rectal and pharyngeal) STIs, common among MSM, are mostly asymptomatic and often missed with urethral-only screening. Disclosure of same-sex behaviors by MSM to healthcare providers (HCP) or HCP elicitation of same-sex behaviors through sexual behavioral assessments may facilitate appropriate extragenital STI testing. We describe self-reported prevalence of extragenital STI testing in the past 12 months (p12m) among MSM in the United States stratified by disclosure of same-sex behavior.

Methods Data were obtained from the 2017 American Men's Internet Survey (AMIS), an annual cross-sectional behavioral internet survey of MSM in the United States. We examined the prevalence of MSM who reported any STI test and an extragenital STI test in the $\mathrm{p} 12 \mathrm{~m}$ stratified by disclosure of same-sex behavior among HIV-positive and HIV-negative MSM.

Results Of 10, 049 sexually-active MSM, 42\% reported any STI test and $16 \%$ reported an extragenital STI test in the p12m. Among HIV-negative MSM, 21\% reported an extragenital STI test in p12m; among HIV-positive MSM, 32\% reported an extragenital STI test in the p12m. Ever disclosing same-sex behavior was associated with a higher prevalence of extragenital STI testing $(\mathrm{PR}=1.68 ; 95 \%$ CI 1.53-1.86) among HIV-negative MSM, but not among HIV-positive MSM $(\mathrm{PR}=1.01 ; 95 \%$ CI 0.81-1.25).

Conclusion The prevalence of any STI testing and extragenital STI testing in the $\mathrm{p} 12 \mathrm{~m}$ among MSM was low. To implement STI recommendations, HCPs must be aware of their patients' sexual behaviors. Disclosure of same-sex behaviors by MSM to their HCP or elicitation of these behaviors by HCPs through sexual behavioral assessments could increase STI testing, particularly at relevant anatomic sites of exposure.

Disclosure No significant relationships.

\section{P508 HEPATITIS C REINFECTION RATES AFTER CURE OR CLEARANCE AMONG HIV-INFECTED AND UNINFECTED MEN WHO HAVE SEX WITH MEN}

Carmine Rossi, Zahid Butt, Maryam Darvishian*, Stanley Wong, Amanda Yu, Maria Alvarez, Mel Krajden, Naveed Janjua. BC CDC, Vancouver, Canada

\subsection{6/sextrans-2019-sti.588}

Background Increasing rates hepatitis $\mathrm{C}$ virus (HCV) infection associated with ongoing risk activity have been reported after successful cure or viral clearance. We assessed factors associated with reinfection after treatment-induced or spontaneous clearance (SC) in both HIV-infected and uninfected men who have sex with men (MSM) in British Columbia.

Methods We followed HIV-infected and uninfected MSM who achieved sustained virologic response (SVR) to HCV treatment or had SC with $\geq 1$ subsequent HCV RNA measurement in the British Columbia Hepatitis Testers Cohort. Crude reinfection rates per 100 person-years (PYs) were calculated. Cox regression was used to model adjusted hazard ratios (HRs) and $95 \%$ confidence intervals (CI) for reinfection.

Results We identified 1,349 HCV-infected MSM with SVR $(n=856)$ or SC $(n=493)$, of which $349(26 \%)$ were HIV-positive. HIV-infected MSM were more likely to have histories of injection drug use (41\% vs $21 \%$ ), alcohol use (22\% vs $14 \%$ ) and mental health disorders ( $47 \%$ vs $28 \%$ ), compared to HIV uninfected. A total of 98 reinfections were identified, yielding an overall reinfection rate of 1.9 per 100 PY (1.0 for SVR patients and 2.7 for SC). HIV-infected MSM had higher rates of reinfection (3.1 vs 1.6 per 100 PY) than HIV uninfected individuals. In multivariable analysis, age $<35$ years (HR 3.1, 95\% CI: $1.2,8.1)$, cure through SVR(HR 0.2, 95\% CI: 0.1 , 0.4), HIV infection (HR 2.0, 95\% CI: 1.3, 3.1), problematic alcohol use (HR 2.0, 95\% CI: 1.2, 3.3), injection drug use (HR 2.7, 95\% CI: 1.6, 4.3) and mental health counseling (HR 0.2, 95\% CI: $0.1,0.4$ ) were independently associated with reinfection. Among HIV-infected, injection drug use (HR 1.9, 95\% CI: $0.8,4.2$ ) was less strongly associated with reinfection.

Conclusion Rates of HCV reinfection remain elevated among HIV-infected and uninfected MSM. Substance use is driving reinfection among HIV-negative MSM, while sexual transmission may be more important among HIV-positive MSM.

Disclosure No significant relationships. 